\title{
NUMERICAL QUADRATURES FOR SINGULAR AND HYPERSINGULAR INTEGRALS IN BOUNDARY ELEMENT METHODS*
}

\author{
MICHAEL CARLEY ${ }^{\dagger}$
}

\begin{abstract}
A method is developed for the computation of the weights and nodes of a numerical quadrature which integrates functions containing singularities up to order $1 / x^{2}$, without the requirement to know the coefficients of the singularities exactly. The work is motivated by the need to evaluate such integrals on boundary elements in potential problems and is a simplification of a previously published method, but with the advantage of handling singularities at the endpoints of the integral. The numerical performance of the method is demonstrated by application to an integral containing logarithmic, first, and second order singularities, characteristic of the problems encountered in integrating a Green's function in boundary element problems. It is found that the quadrature is accurate to $11-12$ decimal places when computed in double precision.
\end{abstract}

Key words. numerical quadrature, hypersingular integrals, Hilbert transform, integral equations, boundary element methods, Legendre polynomials

AMS subject classifications. 65D32, 74S15

DOI. $10.1137 / 060666093$

1. Introduction. Boundary integral methods employing hypersingular integrals have become increasingly popular over recent decades with applications in potential problems such as acoustics [6] and fracture mechanics [13]. Hypersingular integrals arise naturally when it is required to compute the field quantities in such problems, for example, the potential and its gradients, and when specialized techniques for the avoidance of "interior resonance" are used, such as that of Burton and Miller [5]. When such integrals arise, they require special numerical treatment and cannot be evaluated using the standard tools of Gaussian quadrature. Instead, approaches tailored to the problem must be used, which can add considerable complexity to the code. This paper introduces a method for the design of numerical quadrature rules which evaluate hypersingular integrals without requiring a detailed analysis of the integrand. The method is based on that of Kolm and Rokhlin [12], who developed a procedure for the design of such rules, but is considerably simplified by the use of Brandão's approach to finite part integrals [4] and is extended to the case of integrals with endpoint singularities - essential if such a scheme is to be used with boundary elements.

To fix ideas, we assume that we are dealing with a two-dimensional potential problem, such as the Laplace or Helmholtz equation:

$$
\begin{aligned}
\nabla^{2} \phi & =0 \\
\nabla^{2} \phi+k^{2} \phi & =0 .
\end{aligned}
$$

In the case of the Helmholtz equation, the singular behavior of the Green's function will be related to the Green's function of the corresponding Laplace equation $[8$, for example]. The Green's functions for the Laplace equation will have a logarithmic

\footnotetext{
${ }^{*}$ Received by the editors July 26, 2006; accepted for publication (in revised form) December 27, 2006; published electronically May 29, 2007.

http://www.siam.org/journals/sisc/29-3/66609.html

†Department of Mechanical Engineering, University of Bath, Bath BA2 7AY, UK (m.j.carley@ bath.ac.uk).
} 
singularity in the planar and axisymmetric case (where the Green's function is proportional to an elliptic integral) and also in the case of an asymmetric problem in an axisymmetric domain $[3,6]$. In each case, discretization of the boundary of the domain and use of a collocation method give rise to integrals of the form

$$
I(x)=\int_{-1}^{1}(\log |x-t|+f(x, t)) L(t) \mathrm{d} t,
$$

where $t,-1 \leq t \leq 1$, is the local coordinate on an element and $x$ is the local coordinate of the collocation point, which may be on the element, or on some adjacent element. The shape function $L(t)$ is typically a polynomial of order up to three.

Obviously, numerical integration of the $\log |x-t|$ term will require that the singular behavior be handled correctly, but since the logarithmic singularity is integrable, this does not present great difficulties; indeed, quadratures for such a problem exist [18]. If, however, it is required to evaluate first, or higher, derivatives of $I$, the integral is improper and more advanced approaches are required. Such integrals arise in the calculation of acoustic scattering by thin bodies [16], due to the "thin-shape breakdown" of the Helmholtz equation, when the Burton and Miller approach is used to avoid spurious resonances [5] and when gradients of the potential are required as, for example, in computing velocities in potential problems in fluid dynamics. It should be noted that in many problems, such as those in axisymmetric domains, $f(x, t)$ will contain logarithmic terms of the form $(x-t) \log |x-t|$ which are exposed by differentiation so that the integrand will contain singularities of more than one order.

The resulting integrals are of the form

$$
\frac{\mathrm{d} I}{\mathrm{~d} x}=\int_{-1}^{1} \frac{L(t)}{(x-t)}+L(t) g(x, t) \mathrm{d} t
$$

and

$$
\frac{\mathrm{d}^{2} I}{\mathrm{~d} x^{2}}=\int_{-1}^{1} \frac{L(t)}{(x-t)^{2}}+L(t) h(x, t) \mathrm{d} t
$$

and must be interpreted as a Cauchy principal value [14, p. 37] and as a Hadamard finite part, respectively [14, p. 31], denoted by $f$ and $f$, when $-1 \leq x \leq 1$. Even when the collocation point $x$ is not on the element but is close to an endpoint, numerical problems arise due to the "offstage singularity" [1], meaning that the integrand is not well approximated by a polynomial.

A number of approaches have been used to handle the strong singularities in the singular and hypersingular integrals. The most obvious is to analyze the integral kernel to extract the singular parts explicitly so that the finite part integral can be evaluated directly [9]. This has the advantage of reliability as the integrand is broken into a singular part or parts and a regular part. The regular part is integrated using Gaussian quadrature and the integrals of the singular part(s) can be interpreted as principal value or finite part integrals using published results [11, for example]. Such an approach is limited, however, in that it requires an analysis of the integrand, i.e., of the kernel of the integral equation, which may be a complicated function not amenable to detailed study.

An alternative approach is to use a quadrature rule which evaluates the integral directly without requiring that it be broken into its singular part(s) and a regular remainder. Such quadratures have been reviewed and developed by Monegato [17] 
among others and allow the integration of functions with singularities of order one or two. While this is useful in some applications, it is preferable to be able to integrate functions containing singularities of all orders up to and including two without having to extract them explicitly. An algorithm for the design of such quadrature rules has been presented by Kolm and Rokhklin [12] and allows finite part integrals to be evaluated using only the value of the integrand at each node and without having to analyze the function. This flexibility, however, comes at the price of losing the property of Gaussian quadratures that they integrate exactly all polynomials up to order $2 N-1$, where $N$ is the number of points in the quadrature rule. In practice, this is not a serious drawback as accuracy indistinguishable from machine precision is rapidly attained. In the case of offstage singularities, they use the algorithm of Ma, Rokhlin, and Wandzura [15] to derive a generalized Gaussian quadrature which can be used to integrate singular functions exactly. The design of such quadratures requires the use of Newton's method to solve a nonlinear system of equations. The effectiveness and accuracy of the method were demonstrated by application to integrals containing singularities of various orders up to and including two over a range of $x,-2.002 \leq$ $x \leq 2.002, x \neq \pm 1$.

The method presented in this paper is similar to that of Kolm and Rokhlin [12] but is simpler to implement and includes the important case of a singularity at an endpoint, a case not considered by Kolm and Rokhlin. The simplification comes about through the use of Brandão's approach to finite part integrals [4]. In the original method, the finite part integrals are computed using special quadratures which must be found as part of the calculation; in this paper, standard Gauss-Legendre quadratures are used, simplifying implementation. It should also be noted that the use of the simpler approach to finite part integrals allows the extension of the method to higher order singularities with no modification to the theory - it is not clear how the special quadratures in the Kolm and Rokhlin approach can be generalized to include singularities of order three and higher or, indeed, singularities of noninteger order.

The extension to include endpoint singularities, easily implemented via the alternative approach to finite part integrals, is important because in boundary element calculations there will always be a need to evaluate integrals at the endpoints of elements and the Kolm and Rokhlin method as published is not applicable to these cases.

2. Quadratures. This section introduces a method for the calculation of the weights of quadrature rules which integrate functions with singularities up to second order, evaluating the integrals in the finite part or principal value sense. The technique is based on that of Kolm and Rokhlin [12], who base their rules on the nodes of an $N$-point Gauss-Legendre quadrature and compute the weights by solving a system of equations for the integrals of Legendre polynomials weighted by each of the singularities which must be handled (equation (2.7) in this paper). The integrals of the weighted polynomials are found numerically, using specially computed quadratures, whose weights must be found as part of the algorithm. In this paper, the procedure is simplified by using Brandão's approach to finite part integrals [4], outlined in section 2.1, which allows the finite part integrals to be computed using standard quadratures. This also allows the method to handle functions with endpoint singularities; this is important when boundary elements with collocation points at their ends are used.

2.1. Finite part integrals. The most convenient approach to the evaluation of finite part integrals seems to be that of Brandão [4], who gives a simple method for computing a finite part integral by breaking the integrand into a singular part and a 
regular part which can be integrated exactly using standard Gaussian quadratures:

$$
\begin{aligned}
f_{-1}^{1} \frac{f(t)}{x-t} \mathrm{~d} t & =\int_{-1}^{1} \frac{f(t)-f(x)}{x-t} \mathrm{~d} t+f(x) f_{-1}^{1} \frac{1}{x-t} \mathrm{~d} t \\
f_{-1}^{1} \frac{f(t)}{(x-t)^{2}} \mathrm{~d} t & =\int_{-1}^{1} \frac{f(t)-f(x)+f^{\prime}(x)(x-t)}{(x-t)^{2}} \mathrm{~d} t \\
+ & f(x) f_{-1}^{1} \frac{1}{(x-t)^{2}} \mathrm{~d} t-f^{\prime}(x) f_{-1}^{1} \frac{1}{x-t} \mathrm{~d} t .
\end{aligned}
$$

The finite part integrals are (see also Monegato [17])

$$
\begin{gathered}
f_{-1}^{1} \frac{1}{x-t} \mathrm{~d} t= \begin{cases}\log |(x+1) /(1-x)|, & |x| \neq 1, \\
\pm \log 2, & x= \pm 1,\end{cases} \\
f_{-1}^{1} \frac{1}{(x-t)^{2}} \mathrm{~d} t \\
= \begin{cases}2 /\left(x^{2}-1\right), & |x| \neq 1, \\
-1 / 2, & x= \pm 1 .\end{cases}
\end{gathered}
$$

The formulae given are also valid for $x$ outside the range of integration so that the same approach can also be used for "near-singular" integrals.

2.2. Integrals of Legendre polynomials. A basic tool in the calculation of the quadrature weights in section 2.3 is the evaluation of finite part and principal value integrals of Legendre polynomials, which are the polynomials orthogonal on the range $-1 \leq t \leq 1$ with a unit weight function. The required integrals are listed below. In most cases, these can be found in standard tables [10] or have been published elsewhere [11]. For $x= \pm 1$, the values are derived in the appendix.

$$
\begin{aligned}
& \int_{-1}^{1} P_{n}(t) \mathrm{d} t \quad= \begin{cases}2, & n=0, \\
0, & n \neq 0,\end{cases} \\
& \int_{-1}^{1} P_{n}(t) \log |x-t| \mathrm{d} t=2\left(Q_{n+1}(x)-Q_{n-1}(x)\right) /(2 n+1), \quad|x| \neq 1, \\
& \int_{-1}^{1} P_{2 m}(t) \log | \pm 1-t| \mathrm{d} t \quad=\frac{2}{4^{m}} \sum_{k=0}^{m} \frac{(-1)^{k}(4 m-2 k) !}{k !(2 m-k) !(2 m-2 k+1) !} \\
& \times\left(\log 2-\sum_{q=0}^{m-k} \frac{1}{2 q+1}\right), \\
& (2.3 \mathrm{~d}) \int_{-1}^{1} P_{2 m+1}(t) \log | \pm 1-t| \mathrm{d} t \quad= \pm \frac{1}{4^{m}} \sum_{k=0}^{m} \frac{(-1)^{k}(4 m-2 k+2) !}{k !(2 m-k+1) !(2 m-2 k+2) !} \\
& \times \sum_{q=0}^{m-k} \frac{1}{2 q+1} \\
& f_{-1}^{1} \frac{P_{n}(t)}{x-t} \mathrm{~d} t=P_{n}(x) f_{-1}^{1} \frac{1}{x-t} \mathrm{~d} t+\sum_{j=0}^{M-1} w_{j} \frac{P_{n}\left(t_{j}\right)-P_{n}(x)}{x-t_{j}} \\
& f_{-1}^{1} \frac{P_{n}(t)}{(x-t)^{2}} \mathrm{~d} t=P_{n}(x) f_{-1}^{1} \frac{1}{(x-t)^{2}} \mathrm{~d} t-P_{n}^{\prime}(x) f_{-1}^{1} \frac{1}{x-t} \mathrm{~d} t \\
& +\sum_{j=0}^{M-1} w_{j} \frac{P_{n}\left(t_{j}\right)-P_{n}(x)+P_{n}^{\prime}(x)\left(x-t_{j}\right)}{x-t_{j}}
\end{aligned}
$$


where $Q_{n}(x)$ is the Legendre function of the second kind, and (2.3e) and (2.3f) are Brandão's method for finite part integrals using an $M$-point Gauss-Legendre quadrature rule $\left(t_{j}, w_{j}\right)$.

The derivatives of the Legendre polynomials, required in evaluating the finite part integral, (2.3f), can be found in standard tables [10] for $x \neq 1$ and are derived in the appendix for $x= \pm 1$ :

$$
P_{n}^{\prime}(x)= \begin{cases}n\left(x P_{n}(x)-P_{n-1}(x)\right) /\left(x^{2}-1\right), & |x| \neq 1 \\ n(n+1) / 2, & x=1 \\ -(-1)^{n} n(n+1) / 2, & x=-1\end{cases}
$$

2.3. Quadrature rules. The paper of Kolm and Rokhlin [12] presents an algorithm for the design of quadrature rules for integrals of the form

$$
f(x, t)=A(x, t)+B(t) \log |x-t|+\frac{C(t)}{x-t}+\frac{D(t)}{(x-t)^{2}} .
$$

The $N$-point rule approximates the integral of $f$,

$$
f_{-1}^{1} f(x, t) \mathrm{d} t \approx \sum_{j=0}^{N-1} w_{j} f\left(x, t_{j}\right)
$$

with the weights $w_{i}$ being functions of $x$. The advantage of such a quadrature is that the functions $A, B, C$, and $D$ of (2.5) need not be known explicitly; it is assumed only that they are polynomials of order up to $M-1$. Then they can be represented as sums of Legendre polynomials $P_{n}$, and the requirement for the quadrature rule is that it integrate exactly $P_{i}(t), P_{i}(t) \log |x-t|, P_{i}(t) /(x-t)$, and $P_{i}(t) /(x-t)^{2}$ for $i=0,1, \ldots, M-1$.

The nodes of the rule are chosen to be the nodes of an $N$-point Gauss-Legendre quadrature which can be computed using the method of Davis and Rabinowitz [7, p. 369]. Then the weights are the solution of the system of equations

$$
\left[\psi_{i}\left(x, t_{j}\right)\right]\left[w_{j}\right]=\left[m_{i}\right]
$$

where

$$
\psi_{i}(x, t)= \begin{cases}P_{i}(t), & i=0, \ldots, M-1 \\ P_{i}(t) \log |x-t|, & i=M, \ldots, 2 M-1, \\ P_{i}(t) /(x-t), & i=2 M, \ldots, 3 M-1 \\ P_{i}(t) /(x-t)^{2}, & i=3 M, \ldots, 4 M-1\end{cases}
$$

and

$$
m_{i}(x)=f_{-1}^{1} \psi_{i}(x, t) \mathrm{d} t .
$$

This is essentially the method of Kolm and Rokhlin [12] simplified by the use of Brandão's approach to finite part integrals. Equations (2.3) allow $m_{i}$ to be calculated using Gauss-Legendre quadrature instead of special quadratures for different orders of singularity [12, equations 71-73]. Using this approach to finite part integrals also opens the way to handling higher order singularities, so-called "supersingular" integrals, and to incorporating singularities of noninteger order. Brandão also gives formulae for finite part integrals with endpoint singularities, a case not considered by Kolm and Rokhlin, and these formulae make the inclusion of endpoint singularities no more difficult than the handling of internal or external singularities. 
2.4. Algorithm. Combining the results of sections 2.1-2.3, an algorithm for the computation of an $N$-point quadrature rule for functions containing singularities up to and including second order and polynomials up to order $M-1$ is made up of the following steps:

1. Find the nodes of the $N$-point Gauss-Legendre quadrature, using, for example, the algorithm of Davis and Rabinowitz [7].

2. At each node, compute the functions $\psi_{i}\left(x, t_{j}\right)$ (see (2.8)).

3. Compute the moments $m_{i}$ (see (2.9)), using (2.3) and an $M$-point GaussLegendre quadrature.

4. Solve (2.7) for the weights $w_{j}$.

The $N \times 4 M$ system of equations (2.7) may be well-posed or under- or overdetermined. It is solved in the least-squares sense when overdetermined and in the minimum norm sense when underdetermined, using the appropriate LAPACK solver [2].

3. Numerical performance. As a check on the accuracy of the method, a reference integral

$$
\begin{gathered}
I_{n}(x)=\int_{-1}^{1} t^{n} \log |x-t| \mathrm{d} t \\
I_{2 m}(x)=\frac{1}{2 m+1}\left[\left(1-x^{n+1}\right) \log |1-x|+\left((-1)^{n}+x^{n+1}\right) \log |1+x|\right] \\
-\frac{2}{2 m+1} \sum_{k=0}^{m} \frac{x^{2 k}}{2 m-2 k+1}, \\
I_{2 m+1}(x)=\frac{1}{2 m+2}\left[\left(1-x^{n+1}\right) \log |1-x|+\left((-1)^{n}+x^{n+1}\right) \log |1+x|\right] \\
-\frac{2}{2 m+2} \sum_{k=0}^{m} \frac{x^{2 k+1}}{2 m-2 k+1}
\end{gathered}
$$

was computed, using quadrature rules of the type derived above. This integral was chosen because it has an integrand of the type which is common in boundary element calculations and because it has an analytical solution which can be compared to the numerical results. The analytical solution can also be differentiated to check the quadrature rule when used on higher order singularities. In each case, the quadrature rule was computed in double precision arithmetic. Kolm and Rokhlin [12] note that constructing the quadratures in double precision gives "11 or 12 correct digits" which is sufficient accuracy for most applications.

Figure 3.1 shows the relative error $\epsilon$ for $M=4$ and $n=0,3,5$ as a function of the number of quadrature points $N$, with

$$
\epsilon=\frac{\left(\sum_{i}\left|K_{n}\left(x_{i}\right)-J_{n}\left(x_{i}\right)\right|^{2}\right)^{1 / 2}}{\left(\sum_{i} K_{n}^{2}\left(x_{i}\right)\right)^{1 / 2}},
$$

where $K_{n}(x)$ is the exact value of the integral,

$$
K_{n}(x)=I_{n}(x)+\frac{\mathrm{d} I_{n}}{\mathrm{~d} x}+\frac{\mathrm{d}^{2} I_{n}}{\mathrm{~d} x^{2}}
$$

and $J_{n}(x)$ is the value computed using the quadrature rule. The integrals were evaluated for $x_{i}=-2+0.0125 i, i=0,1, \ldots, 320$, which includes $x_{i}= \pm 1$. The values of 


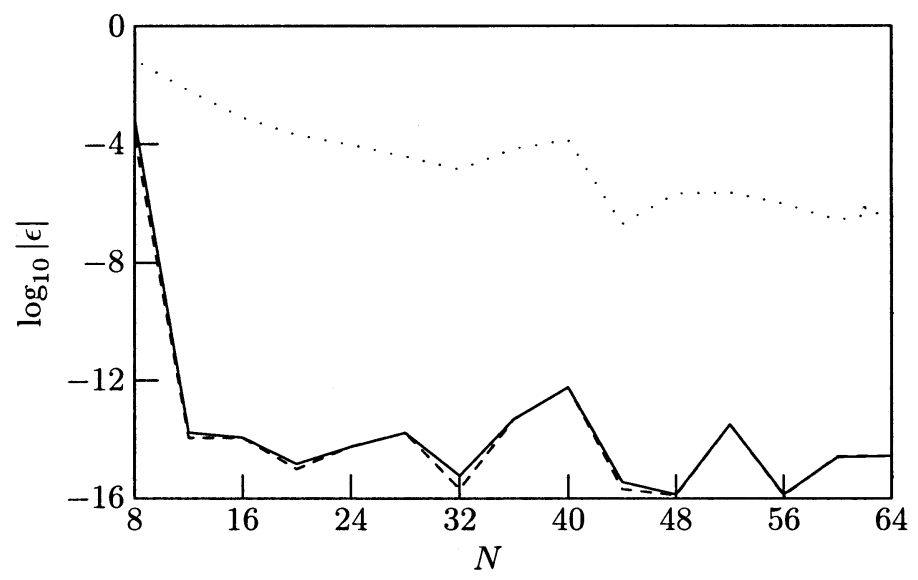

FIG. 3.1. Relative error $\epsilon$ for $M=4, N=12,16, \ldots, 64$, and $n=0,3$, and 5 (solid, dashed, and dotted, respectively).

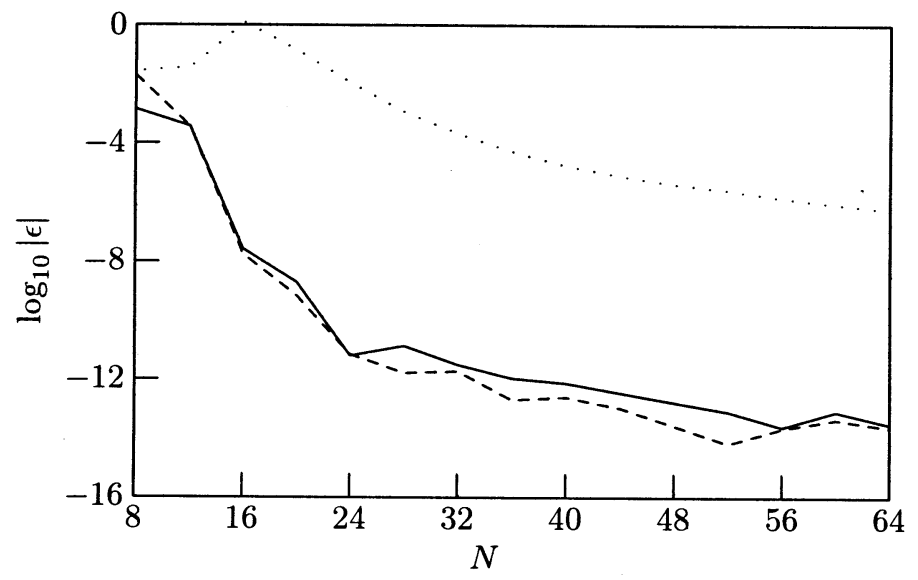

FIG. 3.2. Relative error $\epsilon$ for $M=8, N=12,16, \ldots, 64$, and $n=0,7$, and 11 (solid, dashed, and dotted, respectively).

$n$ were selected as a check on the performance of the quadrature rule for values of $n$ within the selected range of order, i.e., $n \leq M-1$, and to examine the performance of the algorithm for $n$ outside that range. As can be seen, for $n=0,3$ the relative error is quite large $\left(10^{-4}\right)$ for the eight point quadrature but quickly drops to, and remains, less than $10^{-12}$ for $N \geq 12$. The "out of range" function with $n=5$ is never accurately integrated, as would be expected from the definition of the quadrature, showing that care would have to be taken in applications where the Green's function contains terms of higher order such as, for example, in the case of the elliptic integral in axisymmetric potential problems. On the other hand, it is useful to know that the full accuracy of the quadrature is attained at about $N=16$ so that the computational burden of using it is not too great.

Figure 3.2 plots similar data for $M=8$ and $n=0,7,11$. The behavior is similar to that in the $M=4$ case, but an error of $\epsilon \leq 10^{-12}$ is not attained until about $N=24$. Likewise, the error in the $n>M$ case is quite large $(\epsilon \approx 1)$ and does not 
drop below about $10^{-4}$. The most obvious conclusion to be drawn is that the best accuracy of the rule is achieved at about $N=3 M$ and that there is little be gained by increasing $N$ further, unless $M$ is also increased. The results also confirm the conclusion of Kolm and Rokhlin that 11-12 places of accuracy can be achieved when the quadrature weights are computed in double precision.

4. Conclusions. A method has been developed for the derivation of quadrature rules suitable for use in boundary element methods where the integrand has strong singularities up to order two. The technique is a simplification and extension of that of Kolm and Rokhlin [12], allowing for singularities inside and outside the range of integration and at the endpoints, which is an important feature for boundary element methods. The approach has been tested on a reference integral of the type used in boundary element calculations and has been found to be accurate and robust, being accurate to 11-12 places when the quadrature weights are computed in double precision. The approach which has been used is quite general and can be extended to singularities of order higher than two. This will also make the method useful in developing quadratures for three-dimensional boundary element methods.

\section{Appendix. Formulae.}

Most of the formulae used in developing the quadrature technique can be found in standard references [10], but some need to be derived, in particular for the behavior of the Legendre functions at $x= \pm 1$.

The derivative of $P_{n}(x)$ at $x= \pm 1$ can be most readily evaluated from the definition $[10,8.910 .2]$,

$$
P_{n}(x)=\frac{1}{2^{n} n !} \frac{\mathrm{d}^{n}}{\mathrm{~d} x^{n}}\left(x^{2}-1\right)^{n},
$$

and the derivative is

$$
P_{n}^{\prime}(x)=\frac{1}{2^{n} n !} \frac{\mathrm{d}^{n+1}}{\mathrm{~d} x^{n+1}}\left(x^{2}-1\right)^{n} .
$$

Application of Leibnitz's rule [10, 0.42] yields

$$
P_{n}^{\prime}(x)=\frac{1}{2^{n} n !} \sum_{q=0}^{n+1}\left(\begin{array}{c}
n+1 \\
q
\end{array}\right) \frac{\mathrm{d}^{n+1-q}}{\mathrm{~d} x^{n+1-q}}(x+1)^{n} \frac{\mathrm{d}^{q}}{\mathrm{~d} x^{q}}(x-1)^{n},
$$

and, since only the term with $q=1$ survives when $x=-1$,

$$
P_{n}^{\prime}(-1)=-(-1)^{n} \frac{n(n+1)}{2} .
$$

Similarly

$$
P_{n}^{\prime}(1)=\frac{n(n+1)}{2}
$$

The second formula required is the integral

$$
\int_{-1}^{1} P_{n}(t) \log |x-t| \mathrm{d} t
$$


for $x= \pm 1$. Standard tables [10] give

$$
\begin{gathered}
\int_{-1}^{1} t^{2 m} \log | \pm 1-t| \mathrm{d} t=\frac{2}{2 m+1}\left(\log 2-\sum_{q=0}^{m} \frac{1}{2 q+1}\right), \\
\int_{-1}^{1} t^{2 m+1} \log | \pm 1-t| \mathrm{d} t \quad=\frac{ \pm 1}{m+1} \sum_{q=0}^{m} \frac{1}{2 q+1}
\end{gathered}
$$

which, upon insertion into the expansion for the Legendre polynomials [10, 8.911.1], yields

$$
\begin{aligned}
\int_{-1}^{1} P_{2 m}(t) \log | \pm 1-t| \mathrm{d} t=\frac{2}{4^{m}} \sum_{k=0}^{m} \frac{(-1)^{k}(4 m-2 k) !}{k !(2 m-k) !(2 m-2 k+1) !} \\
\times\left(\log 2-\sum_{q=0}^{m-k} \frac{1}{2 q+1}\right),
\end{aligned}
$$

(A.8b) $\int_{-1}^{1} P_{2 m+1}(t) \log | \pm 1-t| \mathrm{d} t=\frac{ \pm 1}{4^{m}} \sum_{k=0}^{m} \frac{(-1)^{k}(4 m-2 k+2) !}{k !(2 m-k+1) !(2 m-2 k+2) !}$

$$
\times \sum_{q=0}^{m-k} \frac{1}{2 q+1}
$$

\section{REFERENCES}

[1] F. S. Aстоn, Numerical Methods that Work, Mathematical Association of America, Washington, DC, 1990.

[2] E. Anderson, Z. Bai, C. Bischof, S. Blackford, J. Demmel, J. Dongarra, J. Du Croz, A. Greenbaum, S. Hammarling, A. McKenney, and D. Sorensen, LAPACK Users' Guide, 3rd ed., Software Environ. Tools 9, SIAM, Philadelphia, 1999.

[3] J. BJÖRKBERG AND G. KRISTENSSON, Electromagnetic scattering by a perfectly conducting elliptic disk, Canad. J. Phys., 65 (1987), pp. 723-734.

[4] M. P. BRANDÃO, Improper integrals in theoretical aerodynamics: The problem revisited, AIAA J., 25 (1987), pp. 1258-1260.

[5] A. J. Burton AND G. F. Miller, The application of integral equation methods to the numerical solution of some exterior boundary-value problems, Proc. Roy. Soc. London Ser. A., 323 (1971), pp. 201-210.

[6] M. CARLEY, Scattering by quasi-symmetric pipes, J. Acoustical Society of America, 119 (2006), pp. 817-823.

[7] P. J. Davis and P. Rabinowitz, Methods of Numerical Integration, Academic Press, New York, 1975.

[8] T. W. Dawson, On the singularity of the axially symmetric Helmholtz Green's function, with application to BEM, Appl. Math. Model., 19 (1995), pp. 590-599.

[9] M. H. Dunn, J. Tweed, And F. FArassat, The application of a boundary integral equation method to the prediction of ducted fan engine noise, J. Sound Vibration, 227 (1999), pp. 1019-1048.

[10] I. Gradshteyn and I. M. Ryzhik, Table of Integrals, Series, and Products, 5th ed., Academic Press, London, 1980.

[11] A. C. Kaya AND F. ERdogan, On the solution of integral equations with strongly singular kernels, Quart. Appl. Math., 45 (1987), pp. 105-122.

[12] P. Kolm And V. Rokhlin, Numerical quadratures for singular and hypersingular integrals, Comput. Math. Appl., 41 (2001), pp. 327-352.

[13] A. M. Konsunsky, On the use of interpolative quadratures for hypersingular integrals in fracture mechanics, Proc. Roy. Soc. London Ser. A., 458 (2002), pp. 2721-2733.

[14] M. J. Lighthill, An Introduction to Fourier Analysis and Generalized Functions, Cambridge University Press, Cambridge, UK, 1958. 
[15] J. Ma, V. Rokhlin, And S. Wandzura, Generalized Gaussian quadrature rules for systems of arbitrary functions, SIAM J. Numer. Anal., 33 (1996), pp. 971-996.

[16] R. Martinez, The thin-shape breakdown (TSB) of the Helmholtz integral equation, J. Acoustical Society of America, 90 (1991), pp. 2728-2738.

[17] G. Monegato, Numerical evaluation of hypersingular integrals, J. Comput. Appl. Math., 50 (1994), pp. 9-31.

[18] R. N. L. Smith, Direct Gauss quadrature formulae for logarithmic singularities on isoparametric elements, Engineering Analysis with Boundary Elements, 24 (2000), pp. 161-167. 